\title{
Absorption of Microwaves in Low Intensity Eucalyptus Litter Fire
}

\author{
Douglas Letsholathebe, Kgakgamatso Mphale \\ Physics Department, University of Botswana, Gaborone, Botswana \\ Email: Mphalekm@mopipi.ub.bw
}

Received 13 July 2015; accepted 24 August 2015; published 27 August 2015

Copyright (C) 2015 by authors and Scientific Research Publishing Inc.

This work is licensed under the Creative Commons Attribution International License (CC BY). http://creativecommons.org/licenses/by/4.0/

(c) (7) Open Access

\begin{abstract}
A fuel bed was constructed where various vegetation species could be used as combustion fuel. The fuel bed was equipped with a thermocouple to measure fire temperature and a two-port automatic network analyser to measure microwave scattering parameters in flame medium. The parameters are then used to determine microwave propagation characteristics in fire. The measurements have implications on radio wave communication during wildfire suppression and in remote sensing. The attenuation data also provide an estimation of vegetation fire ionisation and conductivity. Eucalyptus litter fire with a maximum flame temperature of $976 \mathrm{~K}$ was set on the fuel bed and $X$-band microwaves $(7.00-9.50 \mathrm{GHz})$ were caused to propagate through the flame. Attenuation of $0.35-0.90 \mathrm{~dB}$ was measured for microwaves in the frequency range. For the low intensity fire, conductivity was measured to range from $0.00021-0.00055 \mathrm{mho} / \mathrm{m}$ and electron density was to be the range of $1.83-2.24 \times 10^{15} \mathrm{~m}^{-3}$.
\end{abstract}

\section{Keywords}

Wildfire, Microwave Attenuation, Weakly Ionised Gas, Remote Sensing, Electric Conductivity

\section{Introduction}

Vegetation fires are slightly ionised gaseous medium. When the ionised medium is illuminated with microwaves, incumbent electrons are accelerated by electric field set by the incident electromagnetic energy. The electrons undergo repetitive collisions with relatively stationary heavier neutral incumbent flame particles. As they do, they transfer some of the incident electromagnetic energy into the fire medium. We consider a plane polarised electromagnetic wave which traverses a fire plume in $\mathrm{x}$-direction. The electromagnetic energy dissipates such that its electric field strength $(E(x))$ varies as [1];

$$
E(x)=E_{0} \exp \left\{-\int_{0}^{x} \gamma(x) \mathrm{d} x\right\}
$$


where

$$
\gamma=\alpha_{f}+\beta_{f}
$$

is propagation constant, $\alpha_{f}$ and $\beta_{f}$ are attenuation and phase coefficients, respectively. $E_{0}$ is initial electric field strength. The absorption of energy by fire may result in a considerable signal intensity loss for electromagnetic waves propagating it. However, the absorption depends on temperature, type and concentration of metal impurities in the flame [2] [3].

Vegetation contains up to $3.4 \%$ of the element potassium on dry weight basis and a considerable quantity of other alkalis as nutrients [4]. During combustion, organic structure of plants thermally decomposes to release the alkalis and other inorganic particulates into the fire [5]. Thus, vegetation fires are essentially alkali seeded diffusion flames with temperatures up to $1300^{\circ} \mathrm{C}$ [6]. High temperature flames seeded with alkali metal species show high microwave absorption, e.g., in [3] [7]. An explanation for the high absorption is that there is high electron concentration in the flames. Alkalis metals have low ionisation energies, e.g., potassium has ionisation energy of $4.34 \mathrm{eV}$, and are therefore easily ionised in high temperature environments. The absorption increases with the increase in concentration of alkali metal impurities [3] [7], thus high alkali content in plants could make forest fires detrimental to electromagnetic wave communication. In fact, cases of radio wave communication blackout at high frequency (HF) have been reported [8]. Numerical experiments also predict radio wave attenuation coefficients of up $25 \mathrm{~dB} / \mathrm{m}$ in fire environments [9]. However, propagation experiments at super high (SHF) and extreme high (EHF) frequencies above vegetation fire flames, thus in the thermal plume region, have yielded insignificant attenuation [8] [10]. The negligible attenuation observed in [8] [10] could be attributable to cool flame conditions.

In the experiment, propagation measurements were carried out at the hottest region of low intensity eucalyptus litter fire (up to $37 \mathrm{~kW} \cdot \mathrm{m}^{-2}$ ). Attenuation measurements were carried out using a 2-port Automatic Network Analyser. Besides being a direct measure of microwave attenuation by fire, the measurements are important for wildfire-radio wave interaction model validation. The measurements were also used to estimate electrical conductivity of the low intensity vegetation fire and its average electron concentration.

\section{Ionisation in the Fire}

During vegetation combustion, volatile $\mathrm{K}$ and $\mathrm{Na}$ species diffuse and attach to oxygen containing carboxyl groups in charcoal matrix forming organo-alkali structures such as K-O-C [11]. The high combustion rates (up to $100 \mathrm{Ks}^{-1}$ ) combined with forced gas convection of light gases such as hydrogen radical $\left(\mathrm{H}^{+}\right)$through the organic structure facilitate rapid evolution of alkali atoms from the thermally decomposing charcoal into the flame. This occurs when $\mathrm{H}^{+}$and other light volatiles react with the alkali containing carboxyl groups to displace alkali atoms from charcoal attachment sites [11]. The released alkali atoms (e.g., $\mathrm{Na}(\mathrm{g})$ ) are excited by heat and consequently collide with other excited flame particles. This leads to thermal ionization according to the following equation [12]:

$$
\mathrm{FS}(\mathrm{g})+\mathrm{Na}^{*}(\mathrm{~g}) \Leftrightarrow \mathrm{Na}^{+}(\mathrm{g})+\mathrm{FS}(\mathrm{g})+\mathrm{e}^{-}(\mathrm{g})
$$

where: $\mathrm{FS}(\mathrm{g})$ and $\mathrm{Na}^{*}$, are excited flame and sodium particles, respectively; $\mathrm{A}^{+}$and $\mathrm{e}^{-}$are singly ionized alkali particle and an electron, respectively. At normal forest fire temperatures, Vodacek et al. [13] have estimated that about $10 \%-20 \%$ of $\mathrm{K}$ in vegetation is ionized. The thermal ionization process is a dominant electron-producing mechanism in flames seeded with alkalis [12].

A considerable amount of ions in flames are also produced by chemi-ionization reactions [14]. The reaction is associated with the decomposition of metal oxides in fire to produce electrons according to the reaction [15]:

$$
\mathrm{CO}(\mathrm{g})+\mathrm{MO}(\mathrm{g}) \Leftrightarrow \mathrm{M}^{+}+\mathrm{CO}_{2}(\mathrm{~g})+\mathrm{e}^{-}(\mathrm{g})
$$

A considerable amount of chlorine exists in plants. It exists attached to potassium in cell vacuole [16]. In reactions where chlorine is in co-existence with alkali metals such as sodium, it may play a catalytic role in ionization of the latter by hydrogen atoms to produce electrons and metal ions. The process occurs according to the reaction equation [15]:

$$
\mathrm{Na}(\mathrm{g})+\mathrm{H}(\mathrm{g}) \Leftrightarrow \mathrm{Na}^{+}(\mathrm{g})+\mathrm{H}_{2}(\mathrm{~g})+\mathrm{e}^{-}(\mathrm{g})
$$




\section{Microwave Absorption}

Vegetation fires are highly collisional atmospheric pressure plasma [17]. When X-band microwaves illuminate fire, propagation coefficients, $\alpha_{f}$ and $\beta_{f}$, are related to momentum transfer electron-neutral collision frequency $\left(\varphi_{\text {eff }}\right)$ and plume ionisation by the expressions [17]:

$$
\alpha_{f} \cong \frac{\varphi_{\text {eff }}}{2 c}\left[\frac{\omega_{p}^{2}}{\left(\omega^{2}+\varphi_{\text {eff }}^{2}\right)}\right]
$$

and

$$
\beta_{f} \cong \frac{\omega}{c}\left[1+\frac{\omega_{p}^{4}}{8\left(\omega^{2}+\varphi_{e f f}^{2}\right)^{2}} \frac{\varphi_{\text {eff }}^{2}}{\omega^{2}}\right]
$$

where $\omega$, and $c$ are propagation cyclic frequency and speed of light in vacuum, respectively. $\omega_{p}=\left(n e^{2} / m_{e} \varepsilon_{0}\right)^{1 / 2}$ is plasma frequency. $\varepsilon_{0}$ and $m_{e}$ are dielectric permittivity and mass of electron, respectively.

Flame conductivity and ionisation are respectively given by the relations:

$$
\sigma=2 c \varepsilon_{0} \alpha_{f}
$$

and

$$
n=\left\{\alpha_{f} \varphi_{\text {eff }} / 5.292 \times 10^{-6}\right\}
$$

Momentum transfer electron-neutral collision frequency is given by Uhm [18] as:

$$
\varphi_{\text {eff }}=1.36 \times 10^{-12}\left(\frac{T_{r}}{T_{g}}\right)\left(T_{e}\right)^{0.5}
$$

where $T_{r}, T_{g}$ and $T_{e}$ are ambient, flame and electron temperatures respectively. $T_{e}$ for temperatures around 1000 $\mathrm{K}$ is $0.1 \mathrm{eV}$ [19]. Ratio of transmitted microwave amplitude to that of the incident wave is propagation factor $(T)$ and is given by the relation [20];

$$
T=\mathrm{e}^{-i \gamma r}
$$

where $r$ is the length of the path traversed by the electromagnetic beam through the flame. Propagation factor could be measured using the ANA. It is determined by the measuring scattering parameters $S_{11}$ and $S_{21}$. The scattering parameters are related to $T$ by the following relations [20]:

$$
S_{11}=\left[\frac{\Gamma\left(1-T^{2}\right)}{\left(1-\Gamma^{2} T^{2}\right)}\right]
$$

and

$$
S_{12}=\left[\frac{T\left(1-\Gamma^{2}\right)}{\left(1-\Gamma^{2} T^{2}\right)}\right]
$$

where $\Gamma$ is the reflection coefficient given by the expression, $\Gamma=\Omega \pm \sqrt{\Omega^{2}-1}$ where $\Omega=\left(\frac{S_{11}^{2}-S_{21}^{2}+1}{2 S_{11}}\right)$. The sign in the expression for $\Gamma$ is chosen such that $|\Gamma|<1$. Re-arranging (12) and (13), T can be written as:

$$
T=\left[\frac{S_{11}^{2}+S_{21}^{2}-\Gamma}{1-\left(S_{11}+S_{21}\right) \Gamma}\right]
$$

with $T$ determined from S-parameters, the propagation constant $\gamma$ can be worked out from (11), thus;

$$
\gamma=[\ln (1 / T)] / d
$$




\section{Experimental Setup}

\subsection{Combustion Fuel Bed and Automatic Network Analyser}

The equipment used to measure the microwave absorption by fire consisted of a $0.03 \mathrm{~m}$ thick combustion tray, a Hewlett-Packard 8577C Automatic Network Analyser (ANA) with X-band horns and a computer. A layer of river sand, $0.04 \mathrm{~m}$ thick, was put on the tray to protect it from direct heat from combustion. The tray was $1.20 \mathrm{~m}$ long and $0.75 \mathrm{~m}$ wide (Figure 1). The microwaves were generated from ANA, transmitted and received by two X-band transmit-receive horn. The horns were connected to the ANA through the two-port S-parameter test set by coaxial cables. High quality mode transition adapters were used to make the connections between coaxial cables and the horn (see Figure 1).

Eucalyptus (poplar gum) litter of bulk density of $6.03 \mathrm{~kg} \cdot \mathrm{m}^{-3}$ was collected ten (10) days before the experiment and left to dry in a laboratory. This was done to maximise combustion efficiency during burning. The litter was laid over sand in the combustion tray and set alight.

\subsection{Temperature Measurement}

A hole was drilled at the bottom of the combustion tray to allow the insertion of a thermocouple for fire seat temperature measurement. The thermocouple (type K) wire had fibre glass shield which can withstand temperatures up to $723 \mathrm{~K}$. To further insulate the fibreglass shield, the wire was wrapped with a thermal insulation material called Fiberfrax ${ }^{\circledR}$. The thermocouple wire was cut from a $100 \mathrm{~m}$ double braided fiberglass insulated chromel-alumel (24-G/G) thermocouple wire $50 \mu \mathrm{m}$ in diameter. The thermocouple wire was electro-fused at one end to make perfect junction and then tested with a hot air gun and a multimeter. The wire was then fixed to the bottom of the tray by means of a tape and the electro-fused junction was left protruded $0.01 \mathrm{~m}$ above river sand surface. The thermocouple was then wired to PICO ${ }^{\circledR}$ Tech TC-O8 data logger to read in the temperature into a computer throughout each experiment.

\subsection{S-Parameter Measurements}

The ANA was set to sweep from 7.00 to $9.50 \mathrm{GHz}$ logging in 601 S-parameter data points. The analyser was calibrated using Transmit-Reflect-Line (TRL) as in Varadan et al. [21]. The S-parameters were then uploaded to the computer for analysis. The analyser took approximately $2 \mathrm{~s}$ to sample over one sweep, and then there was a latency of about $50 \mathrm{~s}$ before the next sweep could be initiated. Several sweeps and logging of S-parameters were

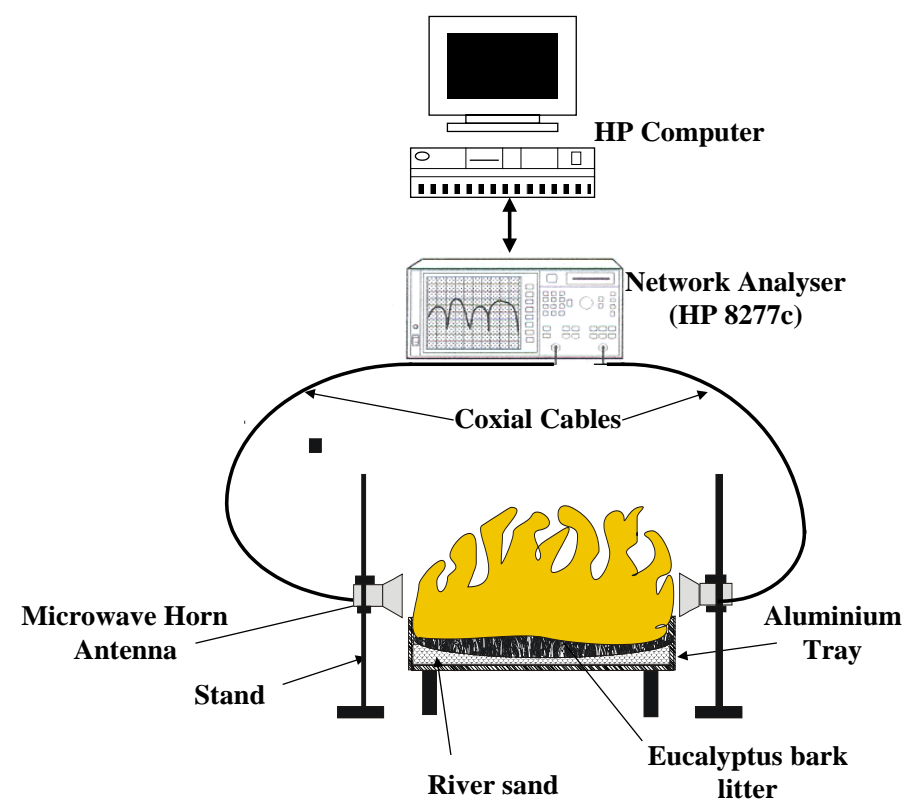

Figure 1. Automatic Network Analyser (ANA) set up for S-parameter measurements. 
carried, e.g., in Figure 2, but those for which flames filled the entire propagation path (by visual inspection) were chosen for S-parameter analysis. Two such cases were els2 and els4 (see Figure 2) which corresponded to the times 178 and 429 s since ignition of the eucalyptus litter.

\section{Experimental Results and Discussions}

\subsection{Fire Temperatures and S-Parameters}

The eucalyptus litter was surface ignited. It took $42 \mathrm{~s}$ for the flame to cover the whole combustion tray. During this time, thermocouple measured temperature rose from an ambient of 302 to $894 \mathrm{~K}$. It took $68 \mathrm{~s}$ for the fire to reach its maximum intensity at which flame temperature was $976 \mathrm{~K}$. Logging of s-parameters was delayed until the fire assumed a steady rate of spread into the fuel layer. This was observed by a constant flame height. After reaching its maximum intensity, its temperature started to decrease steadily to $847 \mathrm{~K}$ after $97 \mathrm{~s}$. At this point the first s-parameter measurement was made (esp1 in Figure 2). The second s-parameter logging was carried out after 178 s (esp2 in Figure 2). Temperature at esp2 was observed to be $834 \mathrm{~K}$. After 201 s, a slight wind deflected the flame such that temperature of the thermocouple was lower than the running average. The effect of the wind lasted over $48 \mathrm{~s}$. Two other such instances occurred during the propagation measurement, one after 90 $\mathrm{s}$ and another after $323 \mathrm{~s}$. They on average lasted for about $60 \mathrm{~s}$. S-parameter measurements, esp3, esp4 and esp5 were made after 281, 429 and $540 \mathrm{~s}$, respectively. Temperatures at the respective periods were, 827, 821 and $817 \mathrm{~K}$. The uploading periods; esp1, esp3 and esp5, were made when the flame covered nearly half of the combustion area and are therefore not used in the analysis. The flame extinguished $107 \mathrm{~s}$ after esp5 and what remained were red hot charcoals.

\subsection{Microwave Attenuation, Electron Density and Conductivity}

Relative microwave absorption at times esp2 and esp4 for frequencies (7.00 - 9.50 GHz) are shown in Figure 3 and Figure 4. The flames at esp2 and esp4 were of slightly different intensity as there was a temperature difference of $13 \mathrm{~K}$. The litter flame at esp2 caused a microwave absorption in the range of $0.90-0.42 \mathrm{~dB}$ while at esp4 suffered a caused a signal loss of $0.72-0.35 \mathrm{~dB}$ for frequency range, respectively. It can be inferred from the measurements that absorption is a factor of rate of alkali emission from fuel. The rate of alkali emissions decreases with the increase in duration of combustion. Thus, the rate of alkali emissions follows mass loss rate curve in thermal decomposition of biomass. Alkali seeding at esp2 is more than at esp4 hence high absorption at esp2 than esp4. Average electron density for esp2 as determined from Equation (23) is $2.24 \times 10^{15} \mathrm{~m}^{-3}$ while at

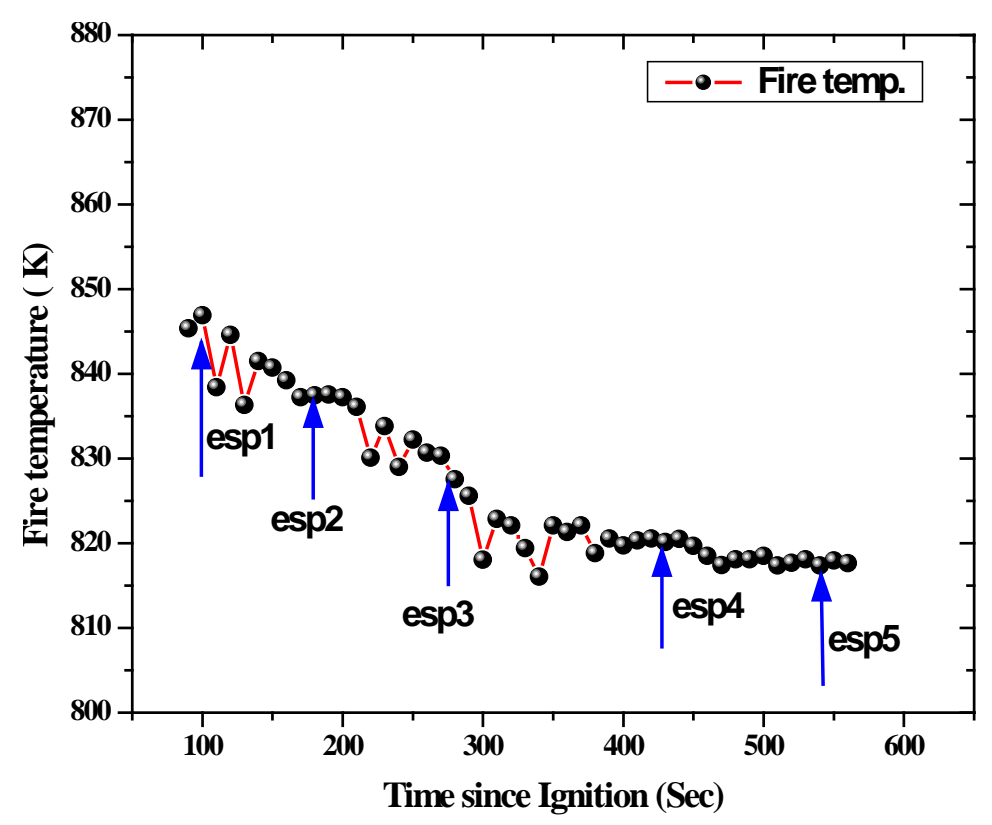

Figure 2. Eucalyptus litter fire temperature during the experiment. 


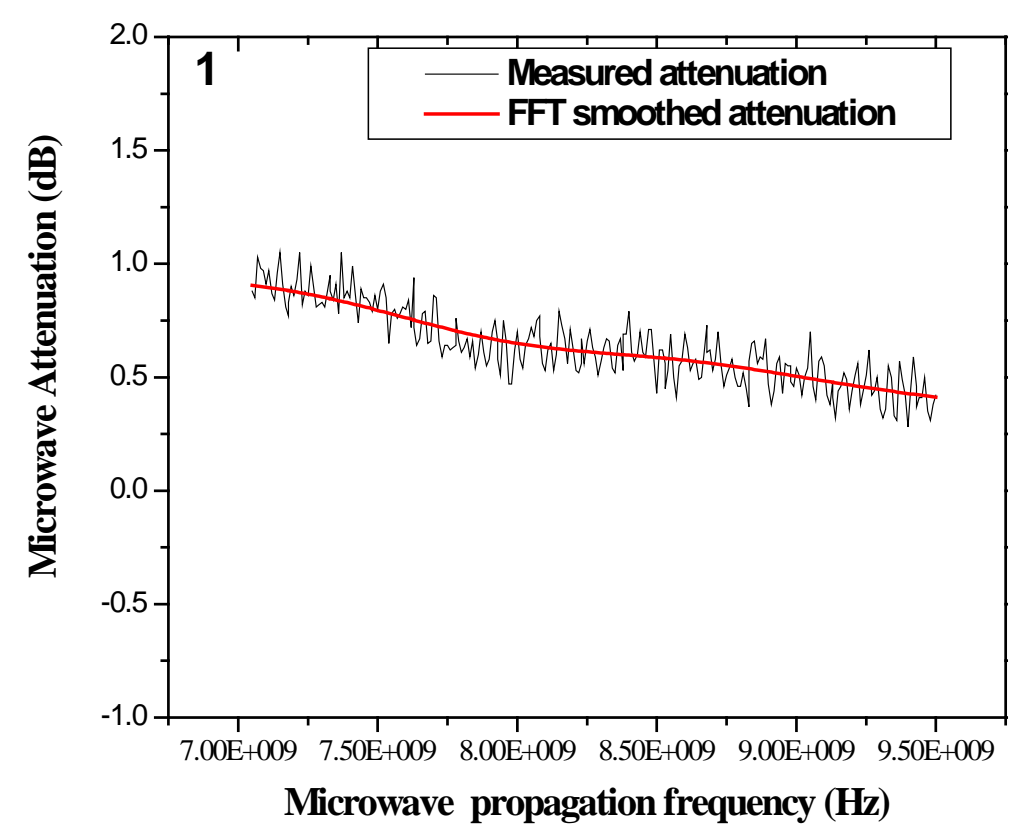

Figure 3. Variation of relative attenuation with propagation frequency at esp2.

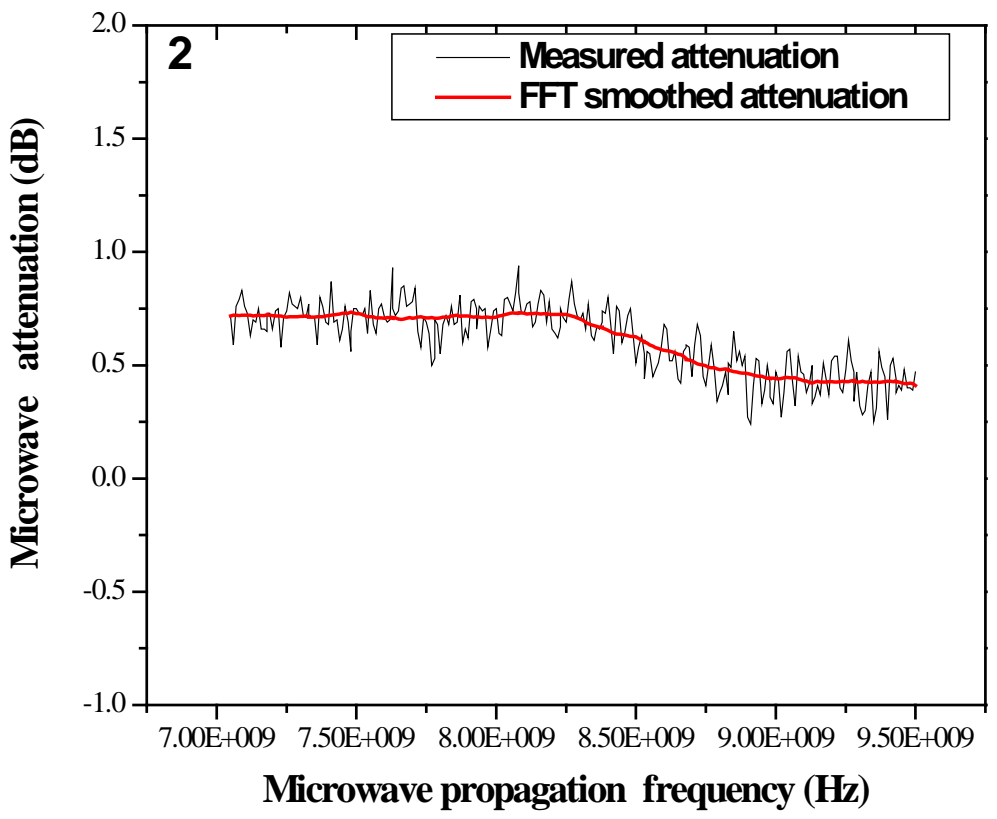

Figure 4. Variation of relative attenuation with propagation frequency at esp4.

esp3 is calculated to be $1.83 \times 10^{15} \mathrm{~m}^{-3}$. Electrical conductivity for the flame at esp2 and esp4 is calculated to have ranges $(2.57-5.50) \times 10^{-4}$ and $(2.11-4.40) \times 10^{-4} \mathrm{mho} / \mathrm{m}$, respectively.

\section{Conclusions}

The electron concentration for the eucalyptus litter fire with maximum radiant heat up to about $37 \mathrm{~kW} \cdot \mathrm{m}^{-2}$ was in the range from $1.83-2.24 \times 10^{15} \mathrm{~m}^{-3}$. Comparatively, the electron densities were lower than those produced from non vegetation fuel flames with much higher temperatures e.g. Belcher et al. [3] and Koretzky et al. [22]. Electron concentration in the flame depends on the type of fuel used as well as its temperature because it is this combination that produces ionisable particles. 
Electrical conductivity ranged from $2.11-5.50 \times 10^{-4} \mathrm{mho} / \mathrm{m}$ for the frequency range considered in the experiment. Typical values of electrical conductivity of pure hydrocarbon flames are low than those measured in the experiment, e.g., in [23]. Maximum, alkali content in eucalyptus is $1.2 \%$. It implies that forest fires that occur in higher alkali contaminated vegetation may seriously affect are Line-of-Sight (LOS) microwave communication systems.

\section{Acknowledgements}

We would like gratefully to acknowledge the Department of Electrical Engineering of James Cook University for providing the equipment for S-parameter measurement. The work is supported by the Staff Development Office of the University of Botswana. It is partly supported by Emergency Management Australia under project No. $60 / 2001$.

\section{References}

[1] Santoru, J. and Gregorie, D.J. (1993) Electromagnetic Wave Absorption in Highly Collisional Plasma. Journal of Applied Physics, 74, 3736-3743. http://dx.doi.org/10.1063/1.354520

[2] Schneider, J. and Hofmann, F.W. (1959) Absorption and Dispersion of Microwaves in Flames. Physical Review, 116, 244-249. http://dx.doi.org/10.1103/physrev.116.244

[3] Belcher, H. and Sudden, T.M. (1950) Studies on the Ionisation Produced by Metallic Salts in Flames II. Reactions Governed by Ionic Equilibrium in Coal-Gas/Air Flames Containing Alkali Metal Salts. Proceedings of the Royal Society of London Series A, 202, 17-39. http://dx.doi.org/10.1098/rspa.1950.0083

[4] Radojevic, M. (2003) Chemistry of Forest Fires and Regional Haze with Emphasis on Southeast Asia. Pure and Applied Geophysics, 12, 157-187. http://dx.doi.org/10.1007/978-3-0348-7970-5 10

[5] Jensen, A.P., Frandsen, F.J., Dam-Johansen, K. and Sander, B. (2000) Experimental Investigation of the Transformation and Release to Gas Phase of Potassium and Chlorine during Straw Pyrolysis Conditions. Energy and Fuels, 11, 1026-1032.

[6] Mphale, K.M., Heron, M. and Verma, T. (2007) Effect of Wildfire Induced Thermal Bubble on Radio Communications. Progress in Electromagnetics Research (PIER), 68, 197-228. http://dx.doi.org/10.2528/pier06072202

[7] Boan, J. (2007) Radio Experiments with Fire. IEEE Antennas Wireless Propagation Letters, 6, 411-414. http://dx.doi.org/10.1109/LAWP.2007.902809

[8] Williams, D.W., Adams, J.S., Batten, J.J., Whitty, G.F. and Richardson, G.T. (1970) Operation Euroka: An Australian Mass Fire Experiment. Report 386, Defense Standards Laboratory, Maribyrnor.

[9] Boan, J. (2006) Radio Communication in Fire Environments. Proceedings of the Wars 2006 Conference, Leura,

[10] Hata, M. and Shigeyuki, D. (1983) Propagation Tests for $23 \mathrm{GHz}$ and $40 \mathrm{GHz}$. IEEE Journal on Selected Areas in Communications, 1, 658-673. http://dx.doi.org/10.1109/JSAC.1983.1145971

[11] Okuno, T., Sonoyama, N., Hayashi, J., Li, C., Sathe, C. and Chiba, T. (2005) Primary Release of Alkali and Alkaline Earth Metallic Species during Pyrolysis of Pulverized Biomass. Energy and Fuels, 19, 2164-2171. http://dx.doi.org/10.1021/ef050002a

[12] Nesterko, N.A. and Taran, E.N. (1971) Ionization and Radiation of Alkali Metals in Acetylene-Air Flame Plasma, with Halogen Additions. Journal of Applied Spectroscopy, 14, 242-244. http://dx.doi.org/10.1007/BF00613182

[13] Vodacek, A., Kremens, R.L., Fordham, S.C., Van Gorden, S.C., Luisi, D., Schott, J.R. and Latham, D.J. (2002) Remote Optical Detection of Biomass Burning Using Potassium Emission Signature. International Journal of Remote Sensing, 23, 2721-2726. http://dx.doi.org/10.1080/01431160110109633

[14] Latham, D. (1999) Space Charge Generated by Wind Tunnel Fires. Atmospheric Research, 51, 267-278. http://dx.doi.org/10.1016/S0169-8095(99)00012-5

[15] Alkemade, M.A. (1979) Fundamentals of Analytical Flame Spectroscopy. Hilger, Bristol.

[16] Bjorkmann, E. and Stromberg, B. (1997) Release of Chlorine from Biomass at Pyrolysis and Gasification Conditions. Energy and Fuels, 11, 1026-1032. http://dx.doi.org/10.1021/ef9700310

[17] Mphale, K.M. and Heron, M. (2008) Nonintrusive Measurement of Ionization in Vegetation Fire Plasma. European Physical Journal: Applied Physics, 41, 157-164. http://dx.doi.org/10.1051/epjap:2008013

[18] Uhm, H.S. (1999) Properties of Plasmas Generated by Electrical Breakdown in Flames. Physics of Plasmas, 6, 43664374. http://dx.doi.org/10.1063/1.873701

[19] Sicha, M. (1979) Measurement of the Electron Energy Distribution Function in a Flame Plasma at Atmospheric Pres- 
sure. Czechoslovak Journal of Physics, 29, 640-645. http://dx.doi.org/10.1007/BF01600014

[20] Kadaba, P.K. (1984) Simultaneous Measurements of Complex Permittivity and Permeability in the Millimeter Region by a Frequency-Domain Technique. IEEE Transactions on Instrumentation and Measurement, 33, 336-347. http://dx.doi.org/10.1109/TIM.1984.4315236

[21] Varadan, V.V., Jose, K.A. and Varadan, V.K. (2000) In Situ Microwave Characterization of Nonplanar Dielectric Objects. IEEE Transactions on Microwave Theory and Techniques, 48, 388-394. http://dx.doi.org/10.1109/22.826837

[22] Koretzsky, E. and Kuo, S.P. (1998) Characterization of an Atmospheric Pressure Plasma Generated by a Plasma Torch Array. Physics of Plasmas, 5, 3774-3780. http://dx.doi.org/10.1063/1.872741

[23] Adler, F.P. (1954) Measurement of Conductivity of a Jet Flame. Journal of Applied Physics, 25, 903-908. http://dx.doi.org/10.1063/1.1721767 